\title{
ORGANIZED BEHAVIORAL RESPONSES TO LATERAL HYPOTHALAMIC ELECTRICAL STIMULATION IN INFANT RATS ${ }^{1}$
}

\author{
TIMOTHY H. MORAN," GARY J. SCHWARTZ, AND ELLIOTT M. BLASS \\ Department of Psychology, Johns Hopkins University, Baltimore, Maryland 21218 \\ Received May 17, 1982; Revised July 29, 1982; Accepted September 15, 1982
}

\begin{abstract}
Brief trains of electrical stimulation (500 msec) were administered to 3-, 6-, 10-, and 15-day-old rat pups through electrodes directed at the medial forebrain bundle (MFB) at the level of the lateral hypothalamus. Stimulation was given at three different frequencies: once per $30 \mathrm{sec}$, once per $20 \mathrm{sec}$, and once per $10 \mathrm{sec}$. Pups 10 days of age and younger became behaviorally activated by stimulation and reliably emitted a series of behavioral responses including mouthing, licking, pawing, gaping, probing, and stretch and lordosis responses. Behavior increased with increasing frequency of stimulation and became more organized with age. Sequences of organized behavior patterns are elicited by MFB stimulation which in their culmination take on the characteristics of motivational responses. Fifteen-day-old pups did not respond to these stimulation parameters. These findings indicate that the components of motivational systems are present and can be elicited at an early age. As development progresses, responses become more organized until stimulation parameters effective in rats 3 to 10 days of age become ineffective in activating behavior in day 15 pups.
\end{abstract}

Intrahypothalamic electrical stimulation induces eating, drinking, gnawing, and a variety of other organized behavioral patterns in adult rats, cats, and other mammals (Margulis and Olds, 1962; Hoebel and Teitelbaum, 1962; Caggiula, 1970; Mogenson and Stevenson, 1966; Roberts and Carey, 1965; Vaughn and Fisher, 1962; MacDonnell and Flynn, 1966; Wise, 1969). These behaviors are stimulation bound in the sense that, following a training period, behavior is elicited reliably during stimulation and, in general, ceases at stimulation offset. The particular behavior pattern elicited depends both upon opportunities provided by the environment for interaction (Valenstein et al., 1968) and, to some extent, upon the specific anatomical locus of stimulation (Wise, 1968). Moreover, in an environment that does not contain any goal objects, stimulation generally is without any obvious behavioral effect (Valenstein et al., 1970).

Hypothalamic stimulation also induces behavior in neonatal rats. Three-day-old rat pups acquired an operant response to receive self-stimulation (Moran et al., 1981). During intrahypothalamic electrical stimulation,

\footnotetext{
${ }^{1}$ This work was supported by Research Grant AM18560 from the National Institute of Arthritis, Metabolism, and Digestive Diseases (E. M. B.).

${ }^{2}$ To whom requests for reprints should be sent at Department of Psychiatry, Johns Hopkins University School of Medicine, Baltimore, MD 21205.
}

pups reliably exhibited behavioral patterns that included mouthing, licking, full body stretches which resembled the behavior of pups during maternal milk letdowns, and lordosis. These behaviors occurred in a warm chamber, bare except for a felt floor. The occurrence of these responses, in the absence of obvious environmental supports, accurately predicted the acquisition of an operant response to obtain self-stimulation.

The present study analyzes, within a developmental framework, the behaviors of rat pups receiving lateral hypothalamic stimulation. A number of specific issues are addressed: How is behavior organized during lateral hypothalamic stimulation in the neonatal rat? Do these responses change in quality and in their interrelationship during development? How do these behaviors differ from ones seen in other classes of stimulation during ontogeny and in brain stimulation in adults?

\section{Materials and Methods}

Subjects. Experimental subjects were the offspring of Sprague-Dawley rats mated and bred in our colony. Females were housed individually 7 to 9 days prepartum in plastic tubs with stainless steel wire lids. Lights in the colony were maintained on a $14 \mathrm{hr}$ on-10 hr off light/ dark cycle, with lights on at 8 A.M. and off at 10 P.M. The colony temperature was maintained at $25^{\circ} \mathrm{C}$ and humidity was not controlled. Food and water were available ad libitum. 
Three-, 6-, 10-, and 15-day-old pups served as experimental subjects. Eight pups per age group were tested. A litter provided a maximum of two pups to an age group, and an individual litter was used only at one age. Pups of both sexes were tested at each age. Weight ranges for each were: day 3,10 to $12 \mathrm{gm}$; day 6,15 to $17 \mathrm{gm}$; day 10, 23 to $26 \mathrm{gm}$; and day 15,32 to $36 \mathrm{gm}$. Thirty minutes prior to surgery, experimental pups were removed from their mother, weighed, and placed with littermates.

Electrode implantation. Electrode units were constructed prior to the time of implantation. A Tefloncoated stainless steel wire $(0.005$ in.) bared at the tip was used for the monopolar electrode and uninsulated stainless steel wire $(0.010 \mathrm{in}$.) served as the skull ground. Both electode and ground were soldered into individual goldplated connectors that were mounted as a unit in a plastic frame. All exposed metal surfaces were coated with Silastic. The skull ground was cut to a length of $0.5 \mathrm{~mm}$. Electrodes were precut to the proper dorsal-ventral coordinate for an individual age.

A Stoelting Co. Stellar stereotaxic device was modified for neonatal surgery by adding an ice bath for use as a surgical stage. The ice bath $(22 \times 12 \times 1.75 \mathrm{~cm}$ high $)$ consisted of a hollowed Plexiglas container with a stainless steel lid. Prongs from the lid extended into the ice to produce a cold surface for maintaining anesthetization of 3- and 6-day-old pups. Ear bars were replaced with concave clamps to fit over the head and ears. A chin support maintained the head in a horizontal position. Three- and 6-day-old pups were anesthetized in ice chips and kept chilled by the cold surgical stage throughout the surgical procedure. To minimize the duration of hypothermia in these younger animals, a littermate served as a model for the construction of an acrylic crown around the electrode unit. Once the acrylic dried, the unit was raised from the head of the model, the model was replaced by the experimental pup, and the electrode unit was lowered into place. Cyanoacrylic cement (Zipbond, Tescom Corp., Minneapolis, MN) was used to anchor the acrylic crown to the skull. This procedure is discussed in greater detail elsewhere (Moran et al., 1981; Moran and Blass, 1982). Ten- and 15-day-old pups were anesthetized by halothane inhalation and implanted as above without using a model.

Electrodes were aimed at the medial forebrain bundle at the level of the lateral hypothalamus. The stereotaxic coordinates for each age, taken from the confluence of the lambdoidal suture and the midsagittal sinus were: day 3: $2.0 \mathrm{~mm}$ anterior $(\mathrm{A}), 1.2 \mathrm{~mm}$ lateral $(\mathrm{L})$, and 6.5 $\mathrm{mm}$ ventral $(\mathrm{V})$ to the skull surface; day $6: 3.3 \mathrm{~mm} \mathrm{~A}, 1.5$ $\mathrm{mm} \mathrm{L}$, and $7.3 \mathrm{~mm} \mathrm{~V}$; day 10: $4.8 \mathrm{~mm} \mathrm{~A}, 1.8 \mathrm{~mm} \mathrm{~L}$, and $7.8 \mathrm{~mm} \mathrm{~V}$; day 15: $5.0 \mathrm{~mm} \mathrm{~A}, 1.8 \mathrm{~mm} \mathrm{~L}$, and $8.3 \mathrm{~mm} \mathrm{~V}$.

Following surgery, pups were placed in an incubator with littermates for $4 \mathrm{hr}$. The temperature in the incubator was maintained at $32^{\circ} \mathrm{C}$ under high humidity. All pups were tested approximately $4 \mathrm{hr}$ after electrode implantation.

Pretest procedure. Three-, 6-, and 10-day-old pups were pretested on a felt-covered surface in a $30^{\circ} \mathrm{C}$ environment to determine the current threshold for stimulation response. A Grass S-44 stimulator with a constant current isolation unit was used to administer $500 \mathrm{msec}$ of pulsed direct current stimulation trains. A train consisted of 30 pulses of a width of 2 msec. Pups underwent a series of test trains in ascending order from 30 to $60 \mu \mathrm{A}$ at $5-\mu \mathrm{A}$ interals. Three pulse trains were applied at each current level separated by a 30-sec delay. The current at which pups responded with mouthing or other oral behaviors to two out of three stimulation trains was used during the testing procedure. Pups that did not respond within this current range were not tested further. Following the pretest, pups were relurned to their littermates in a warm incubator for 20 min before testing. Day 15 pups did not respond during the pretest; therefore, the mean stimulation current for day 10 pups was used for these pups.

Testing procedure. Pups were placed individually on a felt-covered turntable in a glass aquarium maintained at $30^{\circ} \mathrm{C}$ by a heat lamp and humidifier (Hall, 1979a). An open-ended Plexiglas cylinder (diameter, $16.5 \mathrm{~cm}$ for 3 and 6-day-old pups, $19.5 \mathrm{~cm}$ for 10- and 15-day-old pups), placed on the turntable, served as the experimental chamber. Following a 5-min adaptation period, pups' responses to three different stimulation frequencies were recorded on videotape for later analysis. The test session was divided into three 6 -min periods, each separated by 1 min. Pups received stimulation only in minutes 1,3 , and 5 of each period; stimulation was not administered during minutes 2,4 , and 6 . Stimulation was delivered once every $30 \mathrm{sec}$ during the stimulation minutes in the first experimental period, once every $20 \mathrm{sec}$ in the second period, and once every $10 \mathrm{sec}$ in the third period.

Videotaping began $1 \mathrm{~min}$ prior to the initial stimulation period and continued for $2 \mathrm{~min}$ beyond the last stimulation period. If pups continued to exhibit activity, taping was continued $1 \mathrm{~min}$ out of every 5 until behavior returned to base line levels. The output from a time-date generator was superimposed onto the video record. The time of each stimulation was recorded so that the incidence of stimulation was available when the videotapes were scored. The video camera was mounted approximately $2 \mathrm{ft}$. from the test arena such that the testing arena filled the entire image. The turntable was rotated to maintain the pup's head oriented toward the camera at all times to record any oral activity.

Behavioral observations. Tapes were scored using 10sec sampling intervals. The occurrence of all behaviors during each interval was recorded. The behavioral categories were: mouthing, chewing or other oral movements in which the pups did not demonstrate licking or full opening of the mouth; licking, movements of the tongue outside the mouth contacting the felt surface, forepaws, or air; probing, rapid vertical movement of the head with the neck flexed, bringing the snout into contact with the felt substrate; pawing, swiping the snout or head with a forepaw; gaping, raising the head and opening the mouth completely in a slow protracted movement; rolling, turning over onto the side or the back and returning to an upright posture; stretch responses, behavior resembling the responses of pups to maternal milk letdowns, with the forelimbs and hindlimbs extended, raising the body slightly from the felt surface with a straight back; lordosis, behavior resembling an adult lordosis response, with hindlimbs tucked under the body, rump raised, back arched, and the tail deflected to the side; and locomotion, 
movement of any two limbs along the surface whether forward, backward, or laterally. Data were analyzed using an analysis of variance of the absolute number of occurrences of each behavior across stimulation frequencies and age groups.

Histological procedure. Following testing, pups were sacrificed by decapitation and the heads were placed in a glutaraldehyde-based buffered fixative. The skulls of 3and 6-day-old pups were decalcified using a $5 \%$ nitric acid solution and the entire head was mounted in paraffin. The brains of older pups were removed from the skull prior to mounting. Ten-micrometer sections were cut through the hypothalamus and every fifth section was mounted and stained with cresyl violet. Electrode placements were verified microscopically. Pups that did not respond during the pretest also were examined histologically.

\section{Results}

Electrical stimulation of the area of the medial forebrain bundle (MFB) caused a dramatic and reliable change in the behavior of 3-, 6-, and 10-day-old pups. In response to stimulation, young pups exhibited a series of oral and other behaviors which occur in the absence of goal objects or environmental supports. The pattern of responding changed with age and across stimulation frequencies. Some of the prominent behaviors observed in response to stimulation are displayed in Figure 1. This figure shows caricatures of mouthing, licking, probing, pawing, gaping, and stretch and lordosis responses in 3day-old pups. These behaviors were elicited reliably and all behaviors appeared in virtually all of the pups tested.
Day 15 pups were not responsive to this form of stimulation.

In response to stimulation administered once every 30 sec, pups first showed mouthing. As stimulation was continued and increased in frequency, licking ensued, followed by probing, gaping, and other organized behaviors such as stretch and lordosis responses. The pattern of behavioral responses induced by electrical stimulation is shown in Figure 2 for 3-, 6-, and 10-day-old pups. The sequence in which behaviors occurred for individual animals were rank ordered so that the first behavior received a score of 1 , the second a score of 2 , and so on. This response pattern was reliable, $F(4,84)=23.623, p$ $<0.001$, and qualitatively similar in 3-, 6-, and 10-day-old pups, in that there was no significant change in the pattern across ages, $\mathrm{F}(8,84)=1.295, p>0.25$. In contrast, the behavioral pattern of 15-day-old pups differed markedly. The behavior of these pups did not appear to be influenced by the stimulation parameters employed in this experiment. Day 15 pups generally spent a period of time exploring the test arena, followed by a groooming bout, after which they slept for an extended period. Occasionally, pups awakened at some point during the test period and repeated the sequence just described. Stimulation did not affect behavior at this age and behavior was not related in any obvious fashion to stimulation.

\section{Responses to stimulation in 3-, 6-, and 10-day old pups}

Overall Activity. There was a significant increase in the overall occurrence of behavior other than locomotion with increased frequency of stimulation at days 3,6 , and

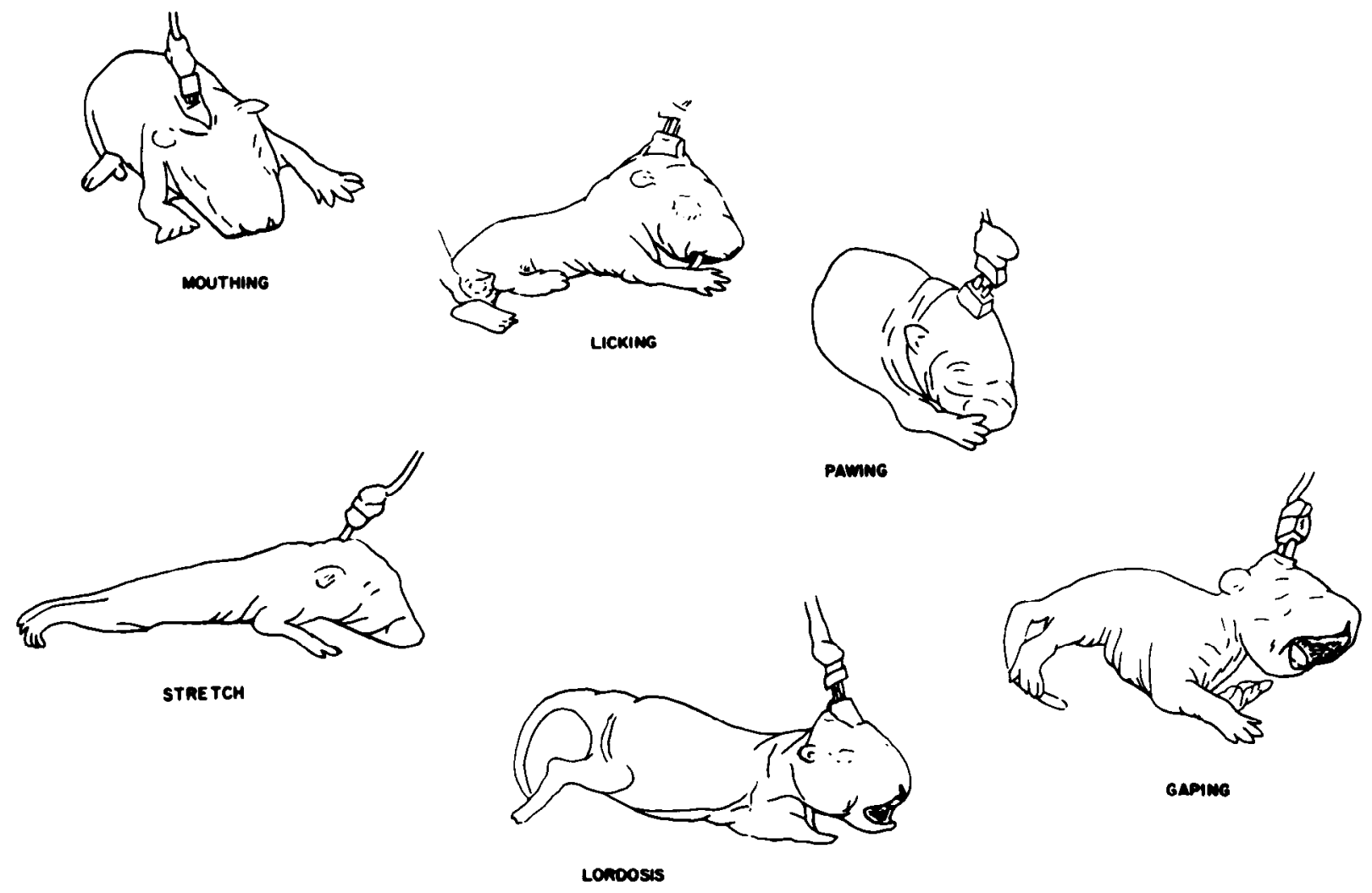

Figure 1. Characteristic behavioral responses to MFB stimulation depicted in a 3-day-old rat pup. 


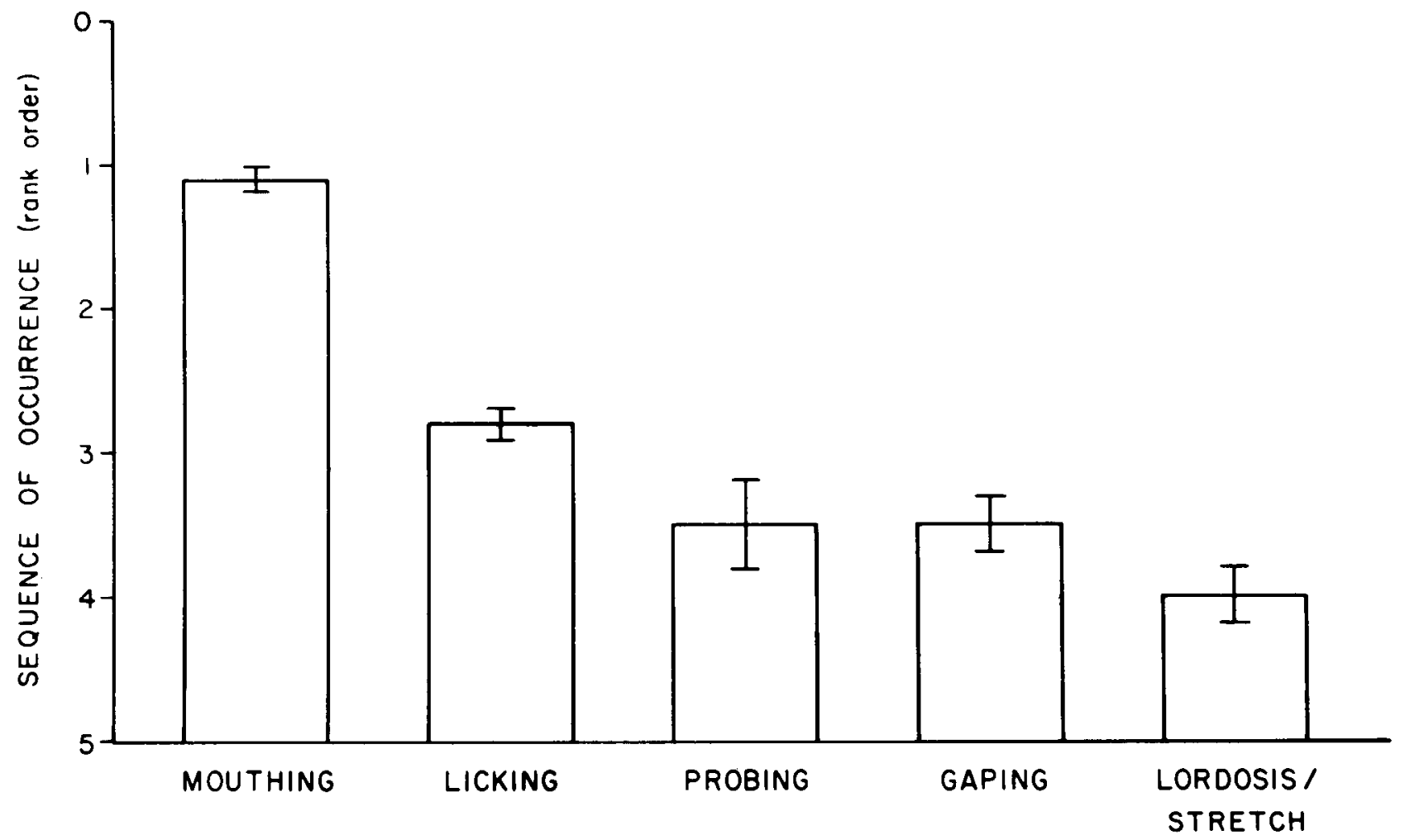

Figure 2. Overall mean \pm SEM ranking of order of occurrence of behavioral responses to MFB stimulation in 3-, 6-, and 10-dayold pups.

10. As stimulation was administered more frequently, from once every $30 \mathrm{sec}$ in the first part of the test to once every $20 \mathrm{sec}$ and finally once every $10 \mathrm{sec}$, the number of behavioral responses increased, $F(2,42)=12.048, p<$ 0.001 ). Locomotion showed the opposite pattern; that is, locomotion decreased with increased stimulation frequency, $\mathrm{F}(2,42)=6.731, p<0.01$. This difference suggests that stimulation affected distinct behaviors differentially, rather than simply increasing the probability of occurrence of all behaviors.

Mouthing. While mouthing, younger pups (3 and 6 days of age) generally assumed a posture with the forepaws extended and the head raised slightly from the surface. As shown in Figure $3 A$, mouthing occurred at a high level throughout the three stimulation frequencies in 3-, 6-, and 10-day-old pups. However, there was a significant increase in the frequency of mouthing across stimulation frequencies, $\mathrm{F}(2,42)=6.894, p<0.01$. There were no significant between-age differences in the number of 10-sec bins in which mouthing occurred.

Licking. Pups frequently would begin to lick the substrate and contact one of their forepaws. When this occurred, pups directed the licking toward the forepaw and brought the paw into contact with the mouth. As shown in Figure $3 B$, licking increased in frequency over stimulation from 4.6 bins at a stimulation frequency of once per $30 \mathrm{sec}$ to $10.8 \mathrm{bins}$ at a stimulation frequency of once per $10 \mathrm{sec}, \mathrm{F}(2,42)=4.059, p<0.05)$. Again, there were no significant differences among age groups in the frequency with which licking occurred.

Probing. Probing in response to stimulation appeared to be qualitatively similar to that seen when suckling (MacFarlane et al., 1982). The occurrence of probing varied across both stimulation frequency and age. Pups of all ages probed but no significant effects were found. Probing coincided with mouthing and licking $66 \%$ of the time, $\mathrm{F}(1,21)=5.749, p<0.05$.

Pawing. As mouthing and licking intensified, pups frequently would raise a paw from the substrate and swipe their heads. Pawing reliably was preceded by mouthing and licking, 84\%, F $(1,23)=44.711, p<0.001$, and rarely occurred in the absence of oral behavior. As shown in Figure $3 C$, pawing increased with increased frequency of stimulation, $\mathrm{F}(2,42)=7.494, p<0.01$. There were no differences in frequency of occurrence across ages from 3 to 10 days of age.

Gaping. Gaping in this setting appeared to represent a heightened level of oral activation. Of the occurrences of gaping, $97 \%$ were preceded within $10 \mathrm{sec}$ by mouthing and licking, $\mathrm{F}(1,23)=51.574, p<0.001$. Occasionally, the tongue extended as the mouth opened. Gaping is an aversive response to noxious taste in adult rats (Grill and Norgren, 1978). While gaping does not appear to be an aversive response in this setting as it was not preceded by backing away and did not involve an arching movement of the back or chin scraping, many adult stimulation sites can invoke both positive and negative responses. Gaping could represent the lowest threshold aversive response at a primarily rewarding site. We do not feel that this is the case, however. Gaping in these experiments appeared qualitatively different from aversive "gaping" responses seen in pups of this age to unpleasant tastes (Hall and Bryan, 1980; P. Kehoe and E. M. Blass, unpublished observation). Gaping was prominent in 3and 6-day-old pups but its incidence decreased in 10-dayolds, $\mathrm{F}(2,21)=9.064, p<0.002$. Figure $3 D$ shows that 

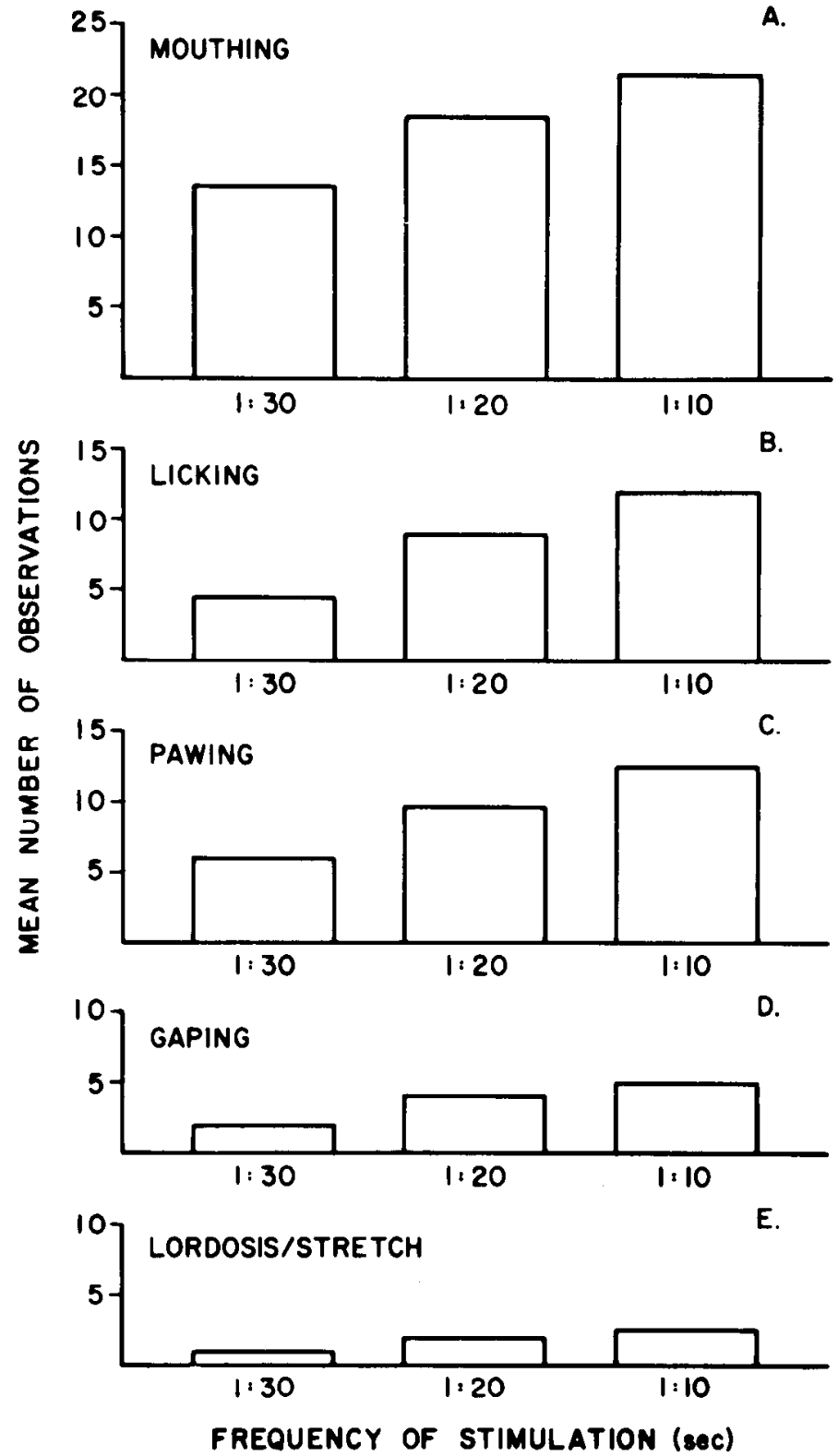

Figure 3. Mean number of sampling intervals in which each behavior was observed at the three stimulation frequencies in 3-, 6-, and 10-day-old pups.

gaping occurred infrequently at low levels of stimulation and increased as stimulation was administered more often, $F(2,42)=3.239, p>0.05$.

Rolling. Rolling occurred when pups became highly activated and was seen primarily in 3-day-olds, $F(2,42)$ $=3.781, p<0.05$. There was a tendency toward an increase in frequency with increasing stimulation at this age but the amount of rolling was highly variable among animals ranging in frequency from 2 bins to over 100 bins. A few of the 3-day-old pups that were very active toward the beginning of the test period exhibited rolling as the primary behavior when receiving stimulation at a high frequency.

Stretch and lordosis responses. These two behaviors have been grouped for the purposes of analysis as they both appear to represent "end behaviors" occurring at the height of behavioral activation. They differ qualitatively in that a stretch response resembles the behavior of pups receiving a maternal milk letdown and lordosis in these young animals resembles an adult lordosis response. These behaviors occurred infrequently at low levels of stimulation and increased as the frequency of stimulation was raised, $\mathrm{F}(2,42)=5.179, p<0.01$. Figure $3 E$ shows this effect. There were no age differences, however, in the quality and pattern of their occurrence across age. As shown in Figure 4, the duration of lordosis, $\mathrm{F}(2,49)=6.872, p<0.003$, and stretch responses, $\mathrm{F}(2,54)$ $=20.418, p<0.001$, increased with increasing age. In 3day-old pups, both lordosis and stretch responses had a high probability of being preceded immediately by oral behaviors. This changed with increasing age. Figure 5 shows that at day 6 and day 10 , the probability of a stretch response occurring within $10 \mathrm{sec}$ of oral responses remained high. However, at day 6 and even more so by day 10 , the probability of a lordosis response being preceded by oral behavior decreased, $p<0.05$ (test for significance of difference between two probabilities). In fact, ear wiggling, another component of adult female sexual behavior, preceded lordosis responses in four of the day 10 pups. Day 10 was the only age at which this behavior was seen, $p<0.05$. Increased duration of these "end behaviors," their segregation with age, and the additional complexity of ear wiggling preceding lordosis start to approximate properties that characterize adult motivated behaviors.

\section{Response to stimulation in 15-day-old pups}

As stated earlier, 15-day-old pups did not show the same behavioral responses to stimulation as younger pups. During the test sessions, 15-day-old pups explored the chamber, groomed, and slept for extended periods. Grooming was a prominent behavior in these animals and did not appear to be linked to stimulation. Grooming can be fractionated into its behavioral components of mouthing, licking, and pawing. When these were counted as individual behavioral occurrences, as shown in Figure 6 , the total number of bins in which active behavior other than locomotion occurred in response to stimulation was still significantly less at day 15 than at any other age, $F(3,28)=8.003, p<0.001$. There were no significant differences between days 3,6 , and 10 even though there did appear to be a trend toward less responsiveness to stimulation with increasing age.

Concordance of behavior with stimulation. The overall temporal pattern of responding to electrical stimulation of the MFB changed with age. Three-day-old pups exhibited a delay in responding to stimulation onset and continued to respond for extended periods following stimulation. With increasing age, behavior coincided more with stimulation until the relationship was lost by day 15. This effect is shown in Figure 7. Since stimulation was administered only in alternate minutes throughout the testing periods, a concordance measure between time of stimulation and time of behavioral responses was generated. Only $50.5+3.1 \%$ of the behavior shown by $3-$ day-old pups occurred within the minutes in which stimulation was administered. This increased to $68.1+1.4 \%$ at day 6 and $73.8+2.4 \%$ at day 10 . There was very little 


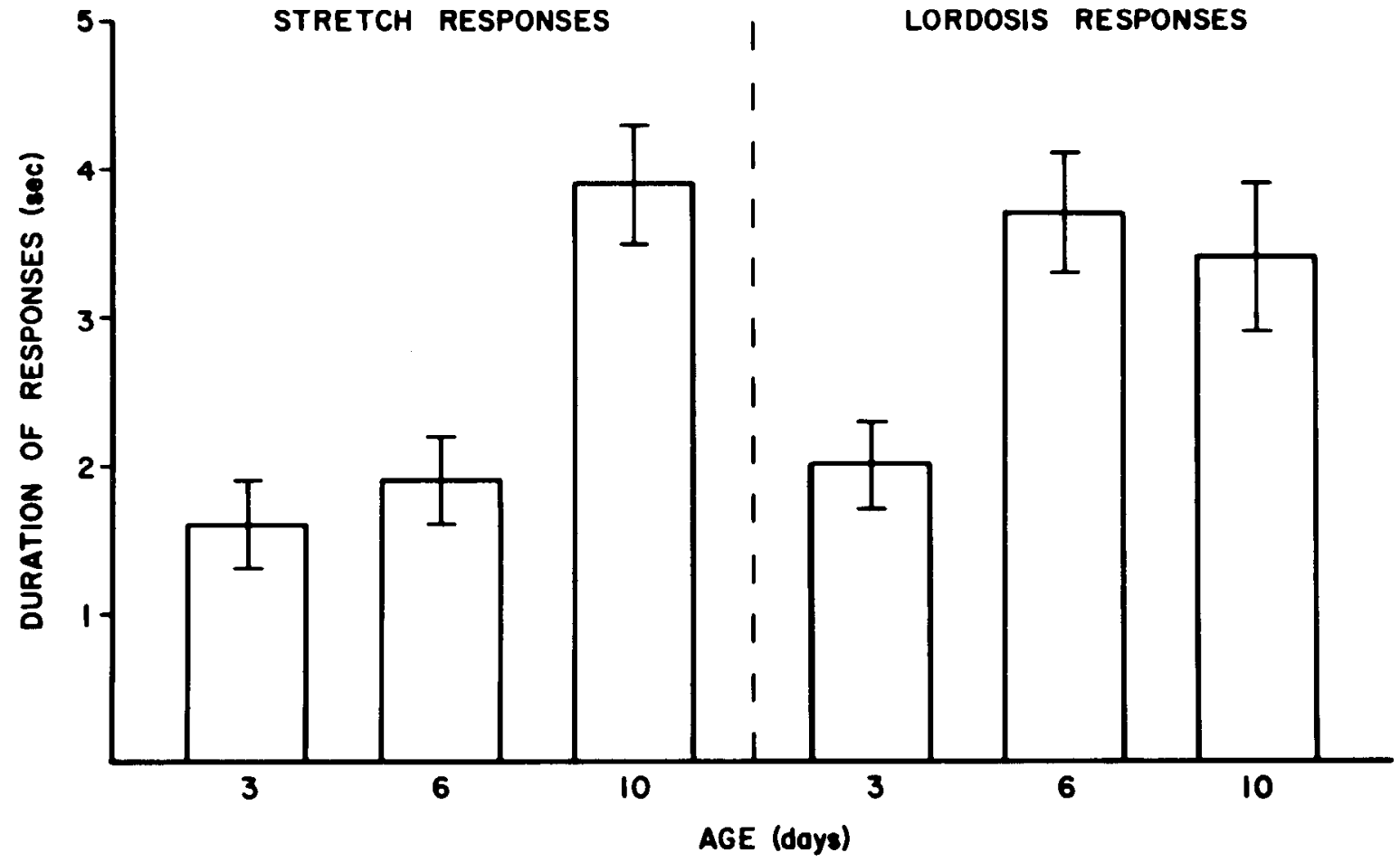

Figure 4. Mean \pm duration (seconds) of stretch and lordosis responses in 3-, 6-, and 10-day-old pups in response to stimulation.

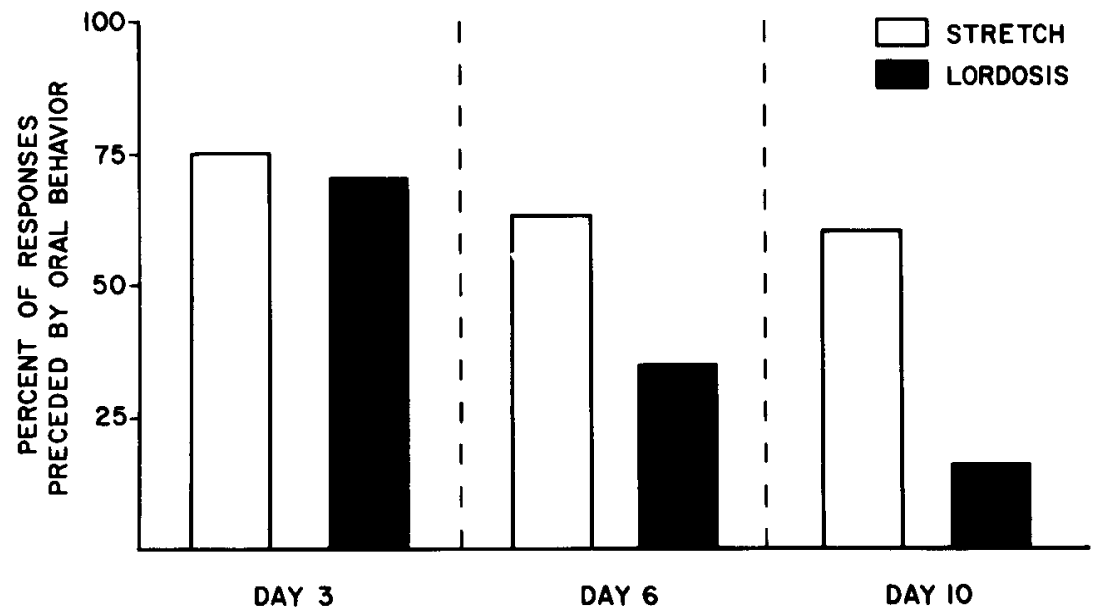

Figure 5. Percentage of stretch or lordosis responses preceded by oral behaviors (mouthing or licking) in 3-, 6-, and 10-day-old pups receiving stimulation.

variability within each age group and differences among all ages are highly significant, $\mathrm{F}(3,28)=9.293, p<0.001$. The trend toward increased coordination of behavior with stimulation was lost at day 15 . The probability of behavior occurring within the stimulation minutes fell to chance levels, $53.9+6.3 \%$, and the variability increased greatly: indeed, unlike day 3 rats, activity was distributed evenly throughout the test period and was not linked to stimulation onset or offset in any apparent fashion. This may be viewed as further evidence of the inability of stimulation of these parameters to induce behavior in 15day-old pups.

Histology. Histological verification of electrode placements revealed that stimulation at sites along the MFB at the level of the hypothalamus was effective in produc- ing stimulation-induced behaviors in pups. The pretest procedure proved to be a highly reliable predictor of responding in the test situation and of electrode placement. Over $95 \%$ of pups that did not meet the criterion of the pretest were found to have placements that were medial or dorsal to the area of the MFB. Only two pups that passed the pretest were later found to have improper placements. The behavior of these pups during the test was lower than the norm and they were omitted from analysis. Both of these placements were medial to the MFB. One impinged on the posterior hypothalamic nuclei. The other punctured the base of the skull and the electrode tip was seated in muscle tissue at the roof of the mouth. This pup showed oral behaviors of licking and mouthing at a low rate throughout the test and did 


\section{TOTAL ACTIVE BEHAVIORS}

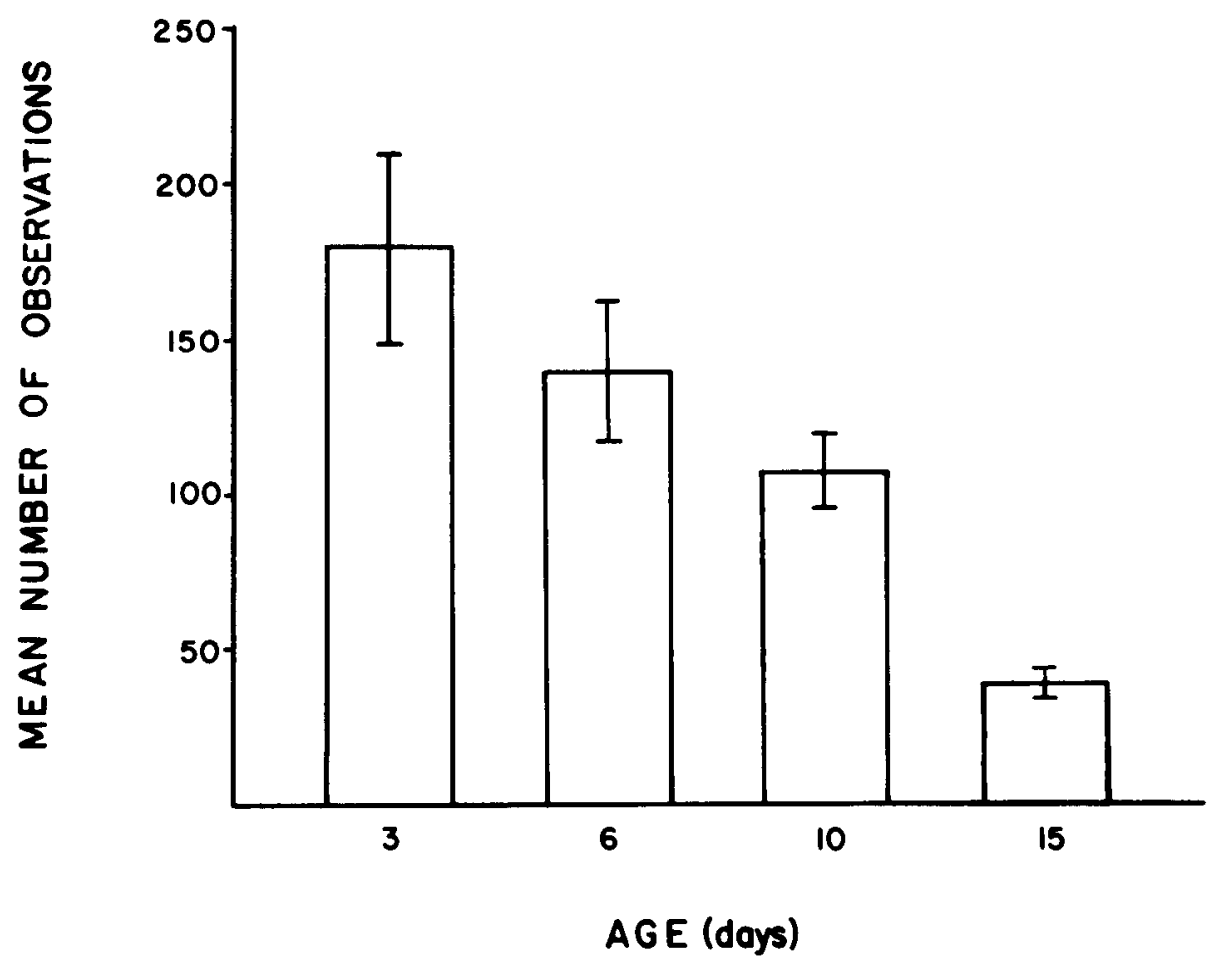

Figure 6. Mean \pm SEM of the number of occurrences of active behaviors in response to stimulation in 3-, 6-, 10-, and 15-day-old pups. Behaviors included in these totals were mothing, licking, probing, pawing, rolling, gaping, stretch responses, and lordosis responses.

\section{CONCORDANCE OF BEHAVIOR \\ WITH STIMULATION}
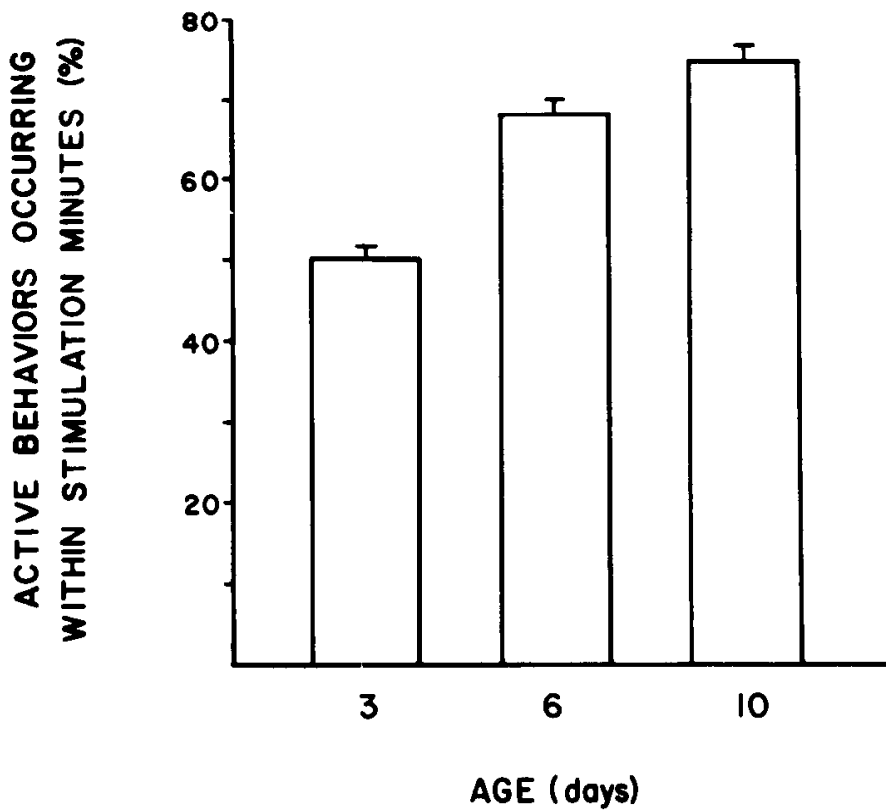

Figure 7. Mean \pm SEM of the percentage of active behaviors which occurred within stimulation minutes in $3-, 6-$, and $10-$ day-old pups. not show the other behaviors with much frequency. As depicted in Figure 8, placements along the medial forebrain bundle anterior or posterior to the lateral hypothalamus within a range of $0.5 \mathrm{~mm}$ were successful. ${ }^{3}$

\section{Discussion}

Following 500-msec stimulation trains to the MFB, pups 3,6 , and 10 days of age display a reliable and orderly series of behaviors which in their culmination take on the characteristics of goal-directed behavior. In response to infrequent stimulation (once per 30 sec), pups assume a posture with the head raised slightly from the felt surface between outstretched forepaws. Pups begin to mouth and at this level of stimulation, the oral behaviors of mouthing and licking predominate. With more frequent stimulation, the incidence of behavior increases and the range of behavior expands to include probing, pawing, and gaping. As pups became even more activated with increasing frequency of stimulation, they frequently present the full body stretch normally seen following a milk letdown. At the height of oral activity, young pups of both genders lordose. Lordosis also was seen frequently in older pups although temporally it was separated from oral behaviors. Prior to lordosis responses, $50 \%$ of the 10 -

\footnotetext{
${ }^{3} \mathrm{~A}$ representative photomicrograph of the electrode tract is shown in Moran et al. (1981).
} 


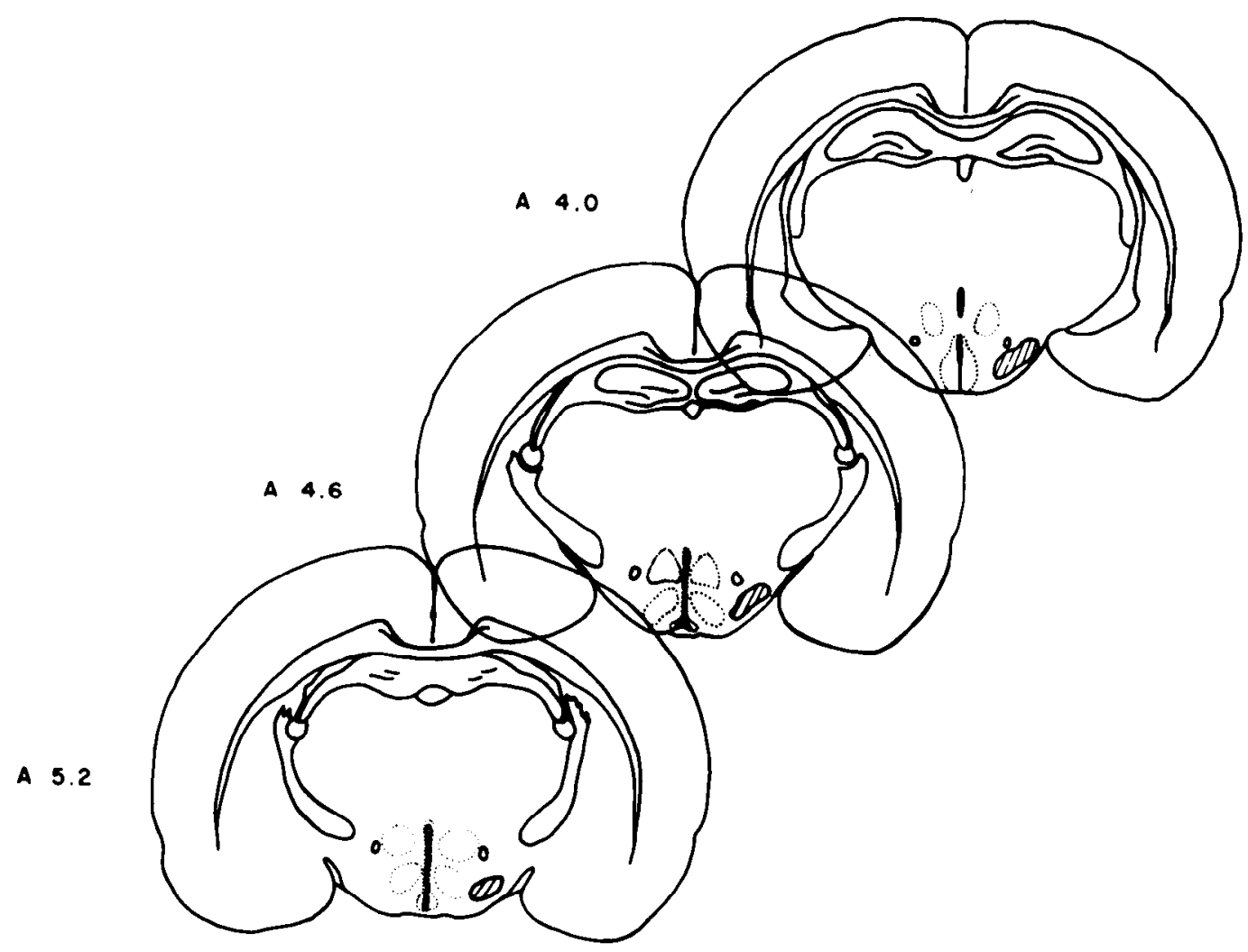

Figure 8. Diagrammatic representations of coronal sections through the hypothalamus illustrating the extent of the area in which stimulation was behaviorally activating (hatched areas). The successful placements follow the ascending tract of the MFB.

day-old pups exhibited ear wiggling, another component of adult female sexual behavior. Once pups were visibly activated, continued stimulation produced nondirected rolling and eliminated other behaviors. By day 15, pups were not obviously activated by these short pulse trains.

A variety of other stimuli also induce behavior in rat pups. Oral infusions of milk in neonatal rats are accompanied by behavioral activation and specific oral responses (Hall, 1979a, b). Pups become highly activated in a warm ambiance especially when independent ingestion of fluid is allowed (Johanson and Hall, 1980; Hall and Bryan, 1980). High levels of activation accompany physical stimulation by the dam in the nest setting (Hofer, et al., 1976; B. MacFarlane and E. M. Blass, unpublished observation), and mimicking this stimulation by stroking pups with a soft artist's brush produces activation (Pedersen and Blass, 1982; Pedersen et al., 1982). Tail pinch in pups 10 days of age and older results in high levels of oral behavior (Szechtman and Hall, 1980).

Yet, the behavioral responses to brain stimulation differ from the behaviors seen in response to some other forms of activating stimulation in the pup. Hall (1979a, b), Hall and Bryan (1980), and Johanson and Hall (1980) have demonstrated that independent ingestion is accompanied by high levels of oral activity. This activation depends on the pup's deprivational status and ambient temperature. When activated by milk, young pups probed, mouthed, locomoted, and rolled over, while older pups directed their behavior toward the nutrient source, exhibiting licking and directed probing. Behavior in this setting was directed toward the nutrient and the activation appeared to be a direct correlate of ingestion. Conversely, brain stimulation initiates and maintains a variety of behavioral responses in the absence of any relevant goal objects. Pups are responsive to brain stimulation through 10 days of age and exhibit more behaviors; that is, lordosis and stretch responses. Behavioral activation accompanying ingestion decreased markedly around 6 days of age even though pups continued to consume milk.

The ontogeny of behavioral responses to brain stimulation differs from the ontogeny of tail pinch-induced behavior in a number of ways. Szechtman and Hall (1980) showed that 5-day-old pups were not responsive to the tail pinch. Responses in 10-day-old pups were slight and it was not until day 15 , the point at which pups do not respond to our parameters of brain stimulation, that pups reliably began to demonstrate oral responses of licking, gnawing, and eating. Thus, behavior appears at a much earlier point in development in response to brain stimulation and the variety of behaviors elicited is much greater.

Behavior induced by brain stimulation in neonates is similar to that in adults in that specific behavioral patterns can be elicited. Again, however, there are important differences. The stimulation used in these experiments was pulse trains of only $500 \mathrm{msec}$ duration, the same stimulation found effective for self-stimulation in neonatal rats (Moran et al., 1981). In untrained adults, stimulation trains of 20 to $30 \mathrm{sec}$ are necessary to induce 
behavior (Wise, 1968; Valenstein et al., 1968). No training period is necessary for behavioral responses in neonates; responses are obtained at the onset of a stimulation session. In adults, behavioral responses to stimulation are stimulation bound; that is, they occur only while the animal is being stimulated. The nature of the pup's responses to stimulation is much less stimulation bound as the onset of behavior may occur up to $30 \mathrm{sec}$ after stimulation initiation and often persists well after stimulation is ended. Behavior does become more closely linked with stimulation in the day 6 and day 10 pups, as demonstrated by the concordance relationships.

Integrated behavioral responses are seen in 3-to 10day-old pups in the absence of environmental supports. This represents the striking difference between neonatal and adult behavior. Adult behavior is not only stimulation bound but stimulus bound as well. Sensory inputs from goal objects such as prey, food, or water are necessary to elicit behavior (MacDonnell and Flynn, 1966; Valenstein et al., 1970). In pups, a variety of behaviors (mouthing, licking, probing, gaping, etc.) are elicited by brain stimulation in the absence of any relevant goal objects. Even such diverse consummatory patterns as stretch and lordosis responses are available to the pup during brain stimulation and do not require specific environmental stimuli for their appearance.

The shift from oral activity to a lordosis response was dramatic in 3-day-old pups. This tendency to display sudden shifts from integrated oral consummatory responses to integrated sexual consummatory responses decreased markedly with age. We see a transition in behavior to a more adultlike pattern. Adult motivational systems are hierarchical in structure; consummatory behaviors that define other motivational states are inhibited and behavior is selective (Gallistel, 1975). In young pups, the expression of behavior elicited by brain stimulation also appears to be determined hierarchically. The controls appear to become more complex with increasing age and presumed neural development; boundaries (Fentress, 1976) between motivational systems become established. We suggest that as a result of increasing neural complexity and behavioral sophistication, hehavior no longer switches from one mode to another at the height of activation and comes to resemble the response patterns of adults to activating stimuli.

The transitions in responding from day 3 to 10 and the lack of responsiveness of 15-day-old rats provide an ontogenic perspective of the organization of motivational processes. Three-day-old rats respond to stimulation with a long latency, but once elicited, responding continues for a considerable time. In fact, many 3-day-old pups continued to respond for up to $20 \mathrm{~min}$ after the test period. Pups highly aroused at early stages of stimulation rolled and lacked behavioral organization to high frequencies of stimulation. These pups regularly began to exhibit the organized behaviors described above after stimulation had ceased and continued to do so for a protracted period. The latency to begin to respond to stimulation decreased with age and, according to the concordance relationship, behavior became progressively more coupled with the stimulation periods.

The data obtained in these experiments suggest that a variety of motivational systems are available in neonatal pups and can be easily elicited by stimulation of the MFB. Organization is advanced to the extent that once oral behaviors are elicited, further activation results in their extension into more complex behaviors. Mouthing and licking precede gaping and stretch responses at all ages. However, organization is not complete as shown by the abrupt shifts from oral behaviors to lordosis. Even at day 10 , when lordosis was preceded by ear wiggling, suggesting strong activation of a sexual system, oral behaviors were seen in the same and preceding 10-sec bins in a substantial number of cases. Behavior becomes more organized and focuses around a particular motivational system for a period of time with increasing age, but not to the complete exclusion of other consummatory patterns. The increased duration of both stretch and lordosis responses with age suggests a greater degree of organization of these "end behaviors" and, possibly, their underlying motivational systems.

The change from behavioral responsiveness in day 10 and younger pups to the lack of responsiveness of 15day-old pups and adults to this brief stimulation suggests the emergence of central inhibitory mechanisms. Although day 15 pups can be trained to self-stimulate to this stimulation (T. H. Moran and G. Schwartz, unpublished observation), such stimulation is no longer behaviorally activating. Day 15 is an age at which many changes are taking place within the CNS of the developing rat. Additional sensory modalities of vision (Gottlieb, 1971; Rose, 1968) and audition (Crowley and Hepp-Raymond, 1966) are maturing. It is at this age that pups show the adult response to tail pinch or tail stimulation. Increased responsiveness to this form of stimulation has been attributed to the development of central dopaminergic pathways (Szechtman and Hall, 1980) and mimics the emergence of apomorphine-induced behavior (Lal and Sourkes, 1973). Cortical inhibitory mechanisms develop and behavioral changes to spreading depression begin to appear which were absent in younger animals (Hicks and D'Amato, 1975). Possibly, the emergence of cortical inhibitory mechanisms, development of sensory modalities, increased development of catecholamine pathways, or a combination of these factors inhibit continued behavioral activation to the form of brain stimulation utilized in the current experiments.

In conclusion, the MFB at the level of the lateral hypothalamus can serve as a neural substrate for behavioral activation in rat pups. The results from these experiments provide evidence for a high degree of behavioral complexity even in 3-day-old pups. Diverse organized behavior patterns can be elicited by stimulation along the MFB which in their culmination take on the characteristics of consummatory responses. Motivational systems can be made accessible at an early age through the activation of electrical stimulation. Behavior in response to stimulation becomes more organized with increasing age until behavioral responses are inhibited with increasing sensory input, catecholamine functioning, and neural maturation.

\section{References}

Caggiula, A. R. (1970) Analysis of the copulation-reward properties of posterior hypothalamic stimulation in male rats. J. Comp. Physiol. Psychol. 70: 399-412. 
Crowley, D. E., and M. C. Hepp-Raymond (1966) Development of cochlear function in the ear of the infant rat. J. Comp. Physiol. Psychol. 62: 427-430.

Fentress, J. C. (1976) Dynamic boundaries of patterned behavior: Interaction and self organization. In Growing Points in Ethology, P. P. G. Bateson and R. A. Hinde, eds., pp. 135169, Cambridge University Press, London.

Gallistel, C. R. (1975) Motivation as a central organizing process: The psychological approach to its functional and neurophysiological analysis. Neb. Symp. Motiv. 22: 183-250.

Gottlieb, G. (1971) Ontogenesis of sensory functions in birds and mammals. In The Biopsychology of Development, E. Tobach, L. Aaronson, and E. Shaw, eds., pp. 67-128, Academic Press, New York.

Grill, H. J., and R. Norgren (1978) The taste reactivity test. I. Mimetic responses to gustatory stimuli in neurologically normal rats. Brain Res. 143: 263-279.

Hall, W. G. (1979a) Feeding and behavioral activation in the infant rat. Science 205: 206-209.

Hall, W. G. (1979b) The ontogeny of feeding in rats. I. Ingestive and behavioral responses to oral infusions. J. Comp. Physiol. Psychol. 93: 977-1000.

Hall, W. G., and T. E. Bryan (1980) The ontogeny of feeding in rats. II. Independent ingestive behavior. J. Comp. Physiol. Psychol. 94: 756-766.

Hicks, S. P., and C. J. D'Amato (1975) Motor sensory cortexcorticospinal system and developing locomotion and placing in rats. Am. J. Anat. 143: 1-42.

Hoebel, B. G., and P. Teitelbaum (1962) Hypothalamic control of feeding and self-stimulation. Science 135: 375-376.

Hofer, M. A., H. Shair, and P. Singh (1976) Evidence that maternal ventral skin substances promote suckling in infant rats. Physiol. Behav. 17: 131-136.

Johanson, I. B., and W. G. Hall (1980) The ontogeny of feeding in rats. III. Thermal determinants of early ingestive responding. J. Comp. Physiol. Psychol. 94: 977-992.

Lal, S., and T. Sourkes (1973) Ontogeny of stereotyped behavior induced by apomorphine and amphetamine in the rat. Arch. Int. Pharmacodyn. Ther. 202: 171-182.

MacDonnell, M.F., and J. P. Flynn (1966) Control of sensory fields by stimulation of the hypothalamus. Science 152: 14061408 .
MacFarlane, B., P. E. Pedersen, C. Cornell, and E. M. Blass (1982) Animal Behavior, in press.

Margulis, D. L., and J. Olds (1962) Identical "feeding" and "rewarding" systems in the lateral hypothalamus of rats. Science 135: 374-375.

Mogenson, G. J., and J. A. P. Stevenson (1966) Drinking and self-stimulation with electrical stimulation of the lateral hypothalamus. Physiol. Behav. 1: 252-254.

Moran, T. H., and E. M. Blass (1982) Organized behavioral responses and self-stimulation induced by intrahypothalamic electrical stimulation. In Neural Basis of Feeding and Reward, D. Novin and B. Hoebel, eds., pp. 59-66, Haer press, Brunswick, ME.

Moran, T. H., M. F. Lew, and E. M. Blass (1981) Intracranial self-stimulation in 3-day-old rats. Science 214: 1366-1368.

Pedersen, P. E., and E. M. Blass (1982) Prenatal and postnatal determinants of the first suckling episode in albino rats. Dev. Psychobiol., 15: 349-355.

Pedersen, P. E., C. L. Williams, and E. M. Blass (1982) Classical conditioning of suckling behavior in 3-day-old rats. J. Exp. Psychol., 8: 329-341.

Roberts, W. W., and R. J. Carey (1965) Rewarding effects of performance of gnawing aroused by hypothalamic stimulation in the rat. J. Comp. Physiol. Psychol. 59: 317-324.

Rose, G. H. (1968) Development of visually evoked electrocortical responses in the rat. Dev. Psychobiol. 1: 35-40.

Szechtman, H., and W. G. Hall (1980) Ontogeny of oral behavior induced by tail pinch and electrical stimulation of the tail in rats. J. Comp. Physiol. Psychol. 94: 436-445.

Valenstein, E. S., V. C. Cox, and J. W. Kakolewski (1968) Modification of motivated behavior elicited by electrical stimulation of the hypothalamus. Science 159: 1119-1120.

Valenstein, E. S., V. C. Cox, and J. W. Kakolewski (1970) Reexamination of the role of the hypothalamus in motivation. Psychol. Rev. 77: 16-31.

Vaughn, E., and A. E. Fisher (1962) Male sexual behavior induced by intracranial electrical stimulation. Science 137 : $758-760$.

Wise, R. A. (1968) Hypothalamic motivational systems: Fixed or plastic neural circuits? Science 162: 377-379.

Wise, R. A. (1969) Plasticity of hypothalamic motivational systems. Science 165: 929-930. 